\title{
Expression profiles of miRNAs in polarized macrophages
}

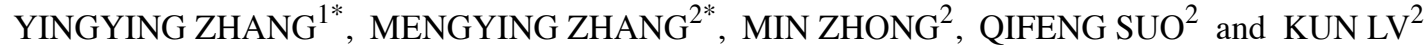 \\ ${ }^{1}$ Laboratory Medicine of Yijishan Hospital; ${ }^{2}$ Central Laboratory of Yijishan Hospital, \\ Wannan Medical College, Wuhu, Anhui 241001, P.R. China
}

Received October 30, 2012; Accepted December 21, 2012

DOI: $10.3892 / \mathrm{jjmm} .2013 .1260$

\begin{abstract}
RNAs (miRNAs) play a crucial role in tissue development and the pathology of various diseases. However, the effects and roles of miRNAs in macrophage polarization have yet to be investigated. In this study, we analyzed and compared the miRNA expression profiles of bone marrowderived macrophages (BMDMs) with two distinct polarizing conditions (classical macrophage activation 'M1' and alternative activation 'M2') using miRNA microarray. In total, 109 miRNAs were differentially expressed between M1 and M2. The differential expression of selected miRNAs was validated by real-time qRT-PCR: miR-181a, miR-155-5p, miR-204-5p and miR-451 were upregulated (fold change $>2, \mathrm{P}<0.05$ ) and miR-125-5p, miR-146a-3p, miR-143-3p and miR-145-5p were downregulated (fold change $<-2, \mathrm{P}<0.05$ ) in $\mathrm{M} 1$ compared with M2. In conclusion, our study may be useful for exploring the precise roles of miRNAs in macrophage differentiation and polarized activation processes in the future.
\end{abstract}

\section{Introduction}

Macrophages are abundant in diverse tissues and organs where they can function as immune effectors, immune regulators, tissue remodelers, or quiescent scavengers. External stimuli can cause macrophages to undergo a marked and coordinated change in the expression of multiple gene products, changing the functional capacity of the cell. The diversity of environments surrounding macrophages in different tissues corresponds to an equally diverse constellation of macrophage phenotypes in the host (1). In the context of specific immune response, the cytokine milieu compels mononuclear phagocytes to express specialized and polarized functional properties. Since they mirror the Th1/Th2 nomenclature, polarized macrophages are regarded as classical macrophage

Correspondence to: Dr Kun Lv, Central Laboratory of Yijishan Hospital, Wannan Medical College, 2 Western Zheshan Road, Wuhu, Anhui 241001, P.R. China

E-mail: lvkun315@126.com

\section{*Contributed equally}

Key words: polarization, bone marrow-derived macrophages, microRNA, microarray activation (known as M1 activation) and alternative activation (known as M2 activation) cells (2-5). Classically polarized activated M1 macrophages have long been known to be induced by IFN- $\gamma$ alone or in combination with microbial stimuli as LPS, or cytokines as TNF- $\alpha$ and GM-CSF. M1 cells have an IL-12 $2^{\text {high }}$, IL-23 $3^{\text {high }}$, IL-10 ${ }^{\text {low }}$ phenotype, are proficient producers of effector molecules (reactive oxygen and nitrogen intermediates) and inflammatory cytokines (IL-1 $\beta$, TNF- $\alpha$ and IL-6), contribute as inducer and effector cells in polarized Th1 responses, and mediate resistance against intracellular parasites and tumors (6-10). By contrast, the alternative M2 form of macrophage activation is a generic name used for various forms of non-classically activated macrophages resulting from cell exposure to IL-4 or IL-13, immune complexes, IL-10, glucocorticoid, or secosteroid (vitamin D3) $(3,9,11)$. The various forms of M2 macrophages share an IL-12 ${ }^{\text {low }}$ and IL-23 ${ }^{\text {low }}$ phenotype, generally exhibit high levels of scavenger, mannose (12), and galactose-type receptors (3), while arginine metabolism is shifted to the production of ornithine and polyamines via arginase (13). However, the regulatory mechanisms controlling the expression of the constellation of genes in macrophages responding to activating conditions are not fully defined.

microRNAs (miRNAs) are small, endogenous non-coding RNA molecules (18-25 nucleotides) that post-transcriptionally regulate gene expression (14). miRNAs identify target mRNA through 5'-seed sequence interactions with miRNA regulatory elements located in the 3'-untranslated region of target mRNA (15). Evidence has indicated that miRNAs play a critical role in regulating cell processes such as cell proliferation, apoptosis, and differentiation (16-18), leading to the hypothesis that miRNAs are partially responsible for the coordinated changes in gene expression occurring during macrophage polarization. This hypothesis is supported by a number of published studies suggesting different miRNAs in the human monocyte/macrophage response to inflammatory stimuli (19-23).

In this study, we employed a miRNA microarray-based profiling assay to document changes in the abundance of miRNAs induced by the activation of primary bone marrowderived macrophages (BMDMs) with two distinct polarizing conditions to span the spectrum of described activation patterns (M1 and M2). Our data revealed that a number of miRNAs were consistently altered under distinct polarizing conditions. Thus, the present study may be useful for exploring the precise roles of miRNAs in macrophage differentiation and polarized activation processes in the future. 


\section{Materials and methods}

Isolation and cultivation of murine BMDMs. Bone marrow cells were obtained by flushing the femurs from Balb/c mice with Dulbecco's modified Eagle's medium (DMEM)-HEPES medium (Gibco, Eggenstein, Germany). Cells were collected in $50 \mathrm{ml}$ tubes and centrifuged for $10 \mathrm{~min}$ at $100 \mathrm{x} \mathrm{g}$. The supernatant was removed, and cells were suspended in

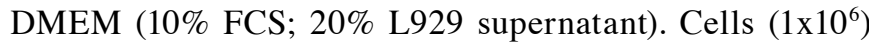
were cultured in 6-well plates at $37^{\circ} \mathrm{C}$ and $5 \% \mathrm{CO}_{2}$ for 7 days (M0). Macrophage polarization was obtained by removing the culture medium and culturing cells for an additional $18 \mathrm{~h}$ in RPMI-1640 supplemented with 5\% FCS and $100 \mathrm{ng} / \mathrm{ml}$ LPS

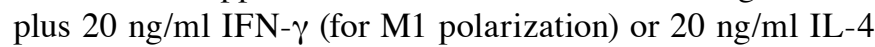
(for M2 polarization).

Arginase activity assay. To prepare cell lysates for the arginase activity assay, cells were first rinsed with ice-cold DPBS twice after each specified treatment and then scraped into $300 \mathrm{ml}$ of lysis buffer containing $50 \mathrm{mM}$ Tris- $\mathrm{HCl}(\mathrm{pH} 7.5), 0.1 \mathrm{mM}$ EDTA, $0.1 \mathrm{mM}$ EGTA, $1 \mathrm{mg} / \mathrm{ml}$ leupeptin, $1 \mathrm{mg} / \mathrm{ml}$ aprotinin, and $0.1 \mathrm{mM}$ phenylmethylsulfonyl fluoride (PMSF; Sigma, St. Louis, MO, USA). Cells were then lysed by sonication at the frequency of $20 \mathrm{kHz}$ (Sonic and Materials, Inc., Danbury, CT, USA) for $30 \mathrm{sec}(10 \mathrm{sec} / \mathrm{cycle})$. Arginase activity in the cell lysates was measured as previously described $(24,25)$. Briefly, cell lysate $(50 \mathrm{ml})$ was added to $50 \mathrm{ml}$ of Tris- $\mathrm{HCl}(50 \mathrm{mM}$; $\mathrm{pH}$ 7.5) containing $10 \mathrm{mM} \mathrm{MnCl}$. Macrophage arginase was then activated by heating this mixture at $55-60^{\circ} \mathrm{C}$ for $10 \mathrm{~min}$. The hydrolysis reaction of L-arginine by arginase was carried out by incubating the mixture containing activated arginase with $50 \mathrm{ml}$ of L-arginine $\left(0.5 \mathrm{M}\right.$; pH 9.7) at $37^{\circ} \mathrm{C}$ for $1 \mathrm{~h}$ and was stopped by adding $400 \mathrm{ml}$ of the acid solution mixture $\left(\mathrm{H}_{2} \mathrm{SO}_{4}: \mathrm{H}_{3} \mathrm{PO}_{4}: \mathrm{H}_{2} \mathrm{O}, 51: 3: 7\right)$. For the colorimetric determination of urea, a-isonitrosopropiophenone $(25 \mathrm{ml}, 9 \%$ in absolute ethanol) was then added, and the mixture was heated at $100^{\circ} \mathrm{C}$ for $45 \mathrm{~min}$. Samples were placed in the dark for $10 \mathrm{~min}$ at room temperature, and the urea concentration was determined spectrophotometrically by the absorbance at $550 \mathrm{~nm}$ measured with a microplate reader (Molecular Devices, Sunnyvale, CA, USA). The amount of urea produced was used as an index for arginase activity.

ELISA assay. Cell culture supernatant was collected and stored at $-80^{\circ} \mathrm{C}$ until assayed. IL-12 and IL-10 concentrations were measured with specific ELISA kits according to the manufacturer's instructions (R\&D Systems, USA).

FACS analysis. BMDMs were stained with the following monoclonal antibodies diluted in 1\% FBS in PBS: FITC anti-F4/80 and PE anti-F4/80 (both from eBioscience Inc., USA), purified anti-inducible nitric oxide synthase (iNOS) (Santa Cruz Biotechnology, Inc., Santa Cruz, CA, USA); PE anti-CD16/32, PE anti-CD206 and PE anti-CD301. Cell fluorescence was measured using FACS analysis and data were analyzed using CellQuest software (all from BD Biosciences, San Jose, CA, USA).

miRNA microarray analysis. The miRNA microarray analysis was performed by the Phalanx Biotech Group (Hsinchu,
Taiwan). Total RNA was extracted from BMDMs using TRIzol reagent (Invitrogen, Burlington, ON, USA) according to the manufacturer's instructions. Total RNA $(2.5 \mu \mathrm{g})$ was labeled with Cy5 fluorescent dyes using a miRNA ULSTM labeling kit (Kreatech Diagnostics, Amsterdam, The Netherlands). Labeled miRNA targets enriched by NanoSep 100K (Pall Corporation, Port Washington, NY, USA) were hybridized to the MRmiOA-mmu-r1-3.0, which contains triplicate 1111 unique miRNA probes from mouse (miRBase Release 17.0), in technical replicates. Following overnight hybridization at $37^{\circ} \mathrm{C}$, non-specific binding targets were washed away, and the slides were dried by centrifugation and scanned using an Axon 4000B scanner. The Cy5 fluorescent intensities of each spot were analyzed by GenePix4.1 software (both from Molecular Devices). The signal intensity of each spot was processed by the R program (version 2.12.1). The fine signals (flag $=0$ ) were extracted and processed by $\log 2$ transformation, and the quantile normalization method and ANOVA test. Experiment data were saved as Microsoft Excel files.

qRT-PCR for miRNAs. Small RNA was extracted from BMDMs as described above. RNA $(2.5 \mu \mathrm{g})$ was then subjected to cDNA synthesis, using either miRNA specific primers or U6 snRNA. Reactions were performed using a TaqMan ${ }^{\circledR}$ MicroRNA reverse transcription kit following the manufacturer's instructions. qRT-PCR was performed using TaqMan ${ }^{\circledR}$ Universal Master Mix on an Applied Biosystems 7500 RealTime PCR Systems. The $20 \mu \mathrm{l}$ PCR reaction mix included $1 \mu \mathrm{l}$ RT products, $10 \mu \mathrm{l}$ TaqMan ${ }^{\circledast}$ Universal PCR Master Mix, No AmpErase ${ }^{\circledR}$ UNG (all from Applied Biosystems, USA), and $1 \mu \mathrm{l}$ TaqMan probe mix. The reactions were incubated in 96-well plates at $95^{\circ} \mathrm{C}$ for $10 \mathrm{~min}$, followed by 40 cycles of $95^{\circ} \mathrm{C}$ for $15 \mathrm{sec}$ and $60^{\circ} \mathrm{C}$ for $1 \mathrm{~min}$. Calculations of miRNA expression levels (M1 vs. M2) were performed using the comparative CT $(\Delta \Delta \mathrm{CT})$ method and normalized against U6 snRNA levels. Reactions were run in triplicate.

Statistical analysis. Data were shown as the mean \pm SEM. Statistical analysis of the data was performed using the two-tailed independent Student's t-test or the ANOVA analysis using the GraphPad Prism (version 4.0) statistical program. $\mathrm{P}<0.05$ was considered statistically significant.

\section{Results}

Identification of ex vivo-programmed M1 and M2 macrophages. To screen for miRNAs whose abundance was altered significantly following incubation of BMDMs in two distinct polarizing conditions, we first generated M1 and M2 macrophages in vitro. M1 macrophages induced by IFN- $\gamma$ and LPS produced a larger number of proinflammatory cytokines IL-12, while M2 macrophages, polarized by IL-4, showed increased IL-10 production (Fig. 1B) and high levels of arginase activity (Fig. 1A). By assessing F4/80 expression, results of the FACS analysis showed that the purity of BMDMs was $\sim 96 \%$ (Fig. 1C). Moreover, FACS analysis showed high levels of iNOS and CD16/32 expression in M1 macrophages and increased CD206 and CD301 expression by M2 macrophages (Fig. 1D). These data confirmed that the polarization conditions used in this study resulted in distinct macrophage phenotypes. 
A

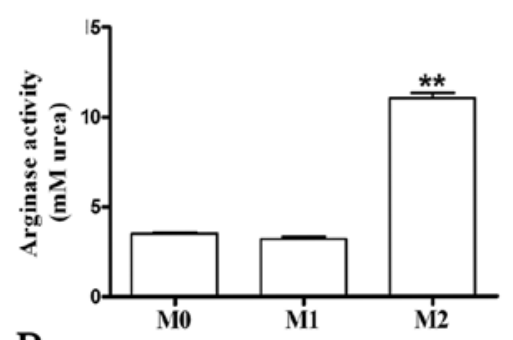

B
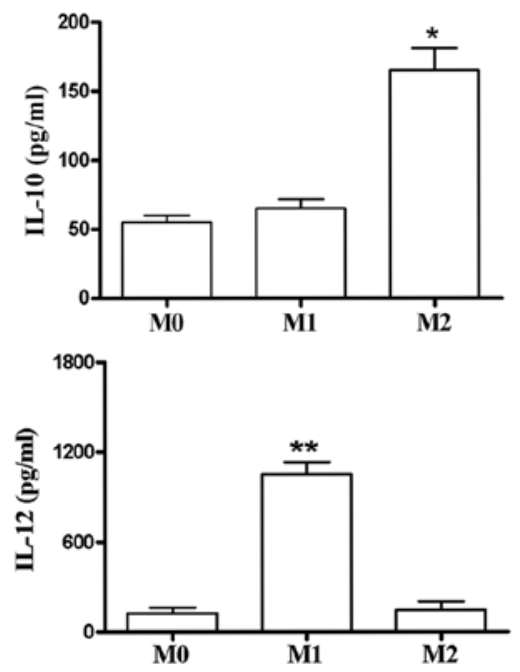

C

D
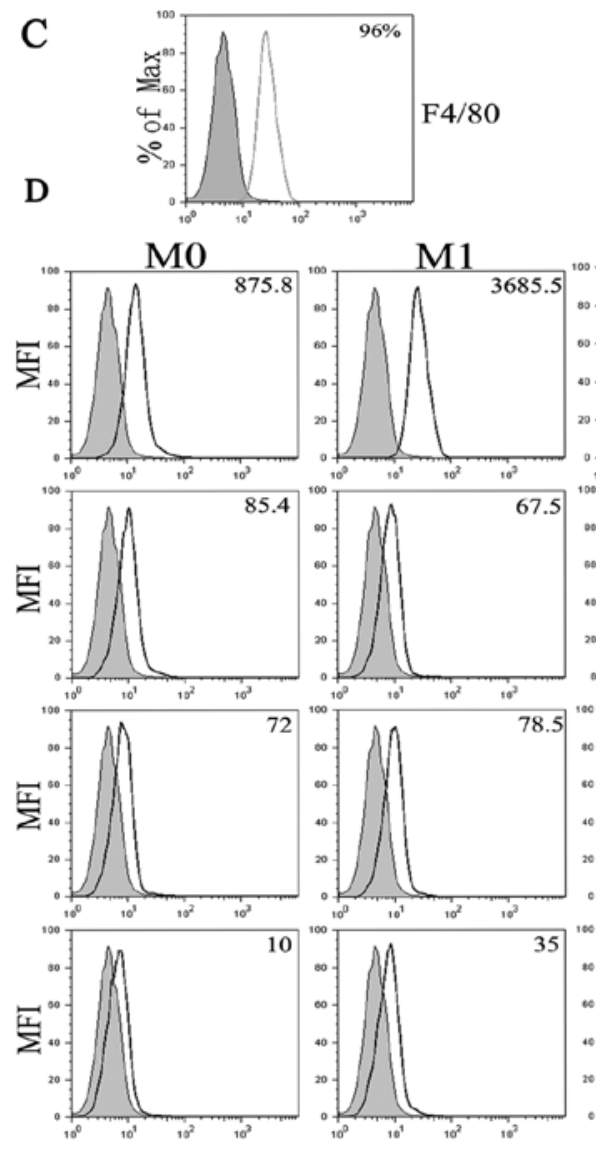
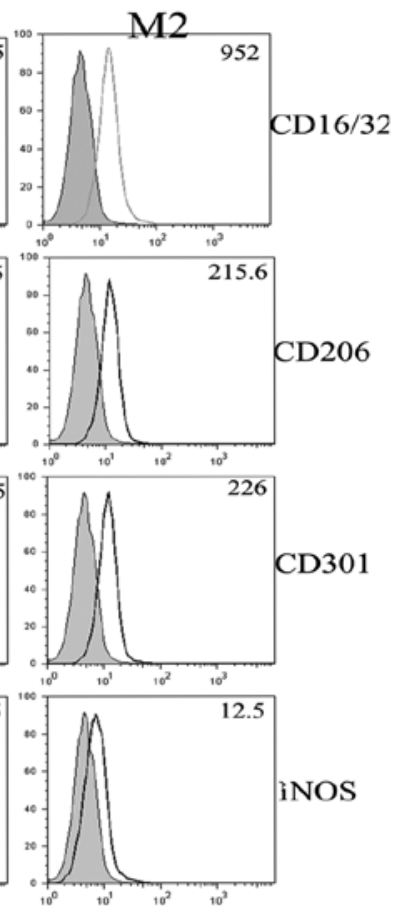

Figure 1. Identification of ex vivo-programmed M1 and M2 macrophages. BMDMs were cultured in the presence of IFN- $\gamma$ (20 ng/ml) plus LPS (100 ng/ml) or IL-4 (20 ng/ml). (A) Arginase activity and (B) IL-12 and IL-10 in the supernatant were assayed. (C) F4/80 and (D) CD16/32, CD206, CD301 and iNOS expression was evaluated by FACS analysis. MFI is the mean fluorescence intensity. Data are representative of three separate experiments, and show the means \pm SEM. ${ }^{*} \mathrm{P}<005 ;{ }^{* *} \mathrm{P}<001$.

miRNA microarray results analysis. To identify miRNAs that are differentially expressed among M0, M1 and M2, we prepared a mouse miRNA microarray containing 1111 oligonucleotide probes complementary to known mammalian miRNAs. Probes were repeated three times in each microarray and each microarray contained controls. The miRNA expression patterns for M1 and M2 were compared. Significance analysis of microarray and a fold change criterion (M1/M2 ratio) of $>2$ or $<0.5$ and $\mathrm{P}<0.05$ were used to identify significant differences. Using these criteria, we identified 109 miRNAs that were differentially expressed between M1 and M2. Of these, 104 miRNAs were upregulated and 5 miRNAs were downregulated in M1 compared with M2 (Fig. 2, Tables I and II).

To evaluate the function categories of miRNAs, we used the bioinformatics tool, TAM (http://202.38.126.151/hmdd/ tools/tam.html) (26), to integrate differentially expressed miRNAs into different sets according to various rules and provide information to mine potential biological meaning behind the list of miRNAs studied. Significantly enriched terms in the miRNAs with increased or decreased expression were apoptosis, cell differentiation, cell proliferation, immune response and inflammation (Table III).

qRT-PCR confirmation of the miRNA microarray results. According to the function classification of differentially expressed miRNAs, sets of miRNAs were selected and
qRT-PCR was used to confirm the results of the miRNA microarray analysis. Of the nine miRNAs identified by the microarray as being the most overexpressed in M1 compared to M2 (miR-155-5p, miR-181a, miR-204-5p, miR-92a, miR221-5p, miR-451, miR-124-3p, miR-25 and miR-127-3p), qRT-PCR confirmed that five (miR-181a, miR-155-5p, miR204-5p, miR-451 and miR-127-3p) were overexpressed (Fig. 3). Of the four miRNAs identified as being underexpressed in M1 by the microarray (miR-125-5p, miR-146a-3p, miR-143-3p and miR-145-5p), the qRT-PCR analysis confirmed that all four were underexpressed (Fig. 3). Overall, the qRT-PCR analysis showed that the miRNA microarray results had some small errors, however, it confirmed that a significant number of miRNAs were differentially regulated in macrophages that responded to $\mathrm{M} 1$ and $\mathrm{M} 2$ polarizing conditions.

\section{Discussion}

Mammalian macrophages are induced to adopt a spectrum of widely divergent phenotypes in response to diverse external stimuli. The present study was based upon the hypothesis that miRNAs are regulators that coordinate, in part, the global changes in the expression of a number of genes that occur after macrophage exposure to different activating conditions.

Expression profiling experiments have reported changes in miRNA expression in human and murine monocytic cells 


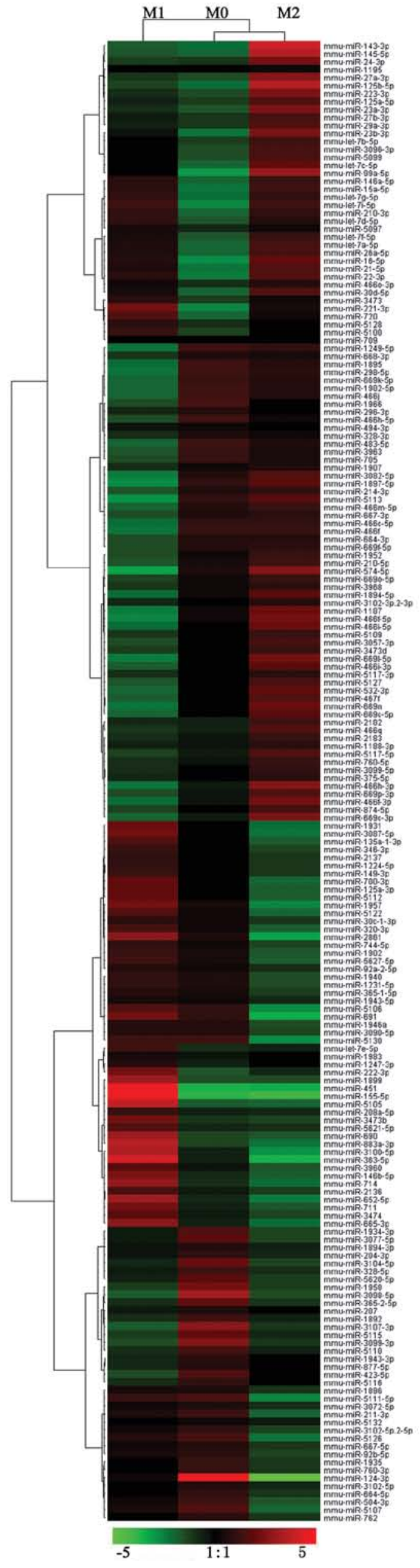

Table I. The number of microRNAs differentially expressed in polarized macrophages (expression fold of $>2$ ).

\begin{tabular}{lccc}
\hline No. & Comparison & Upregulation & Downregulation \\
\hline 1 & M1 vs. M0 & 120 & 1 \\
2 & M2 vs. M0 & 4 & 2 \\
3 & M1 vs. M2 & 104 & 5 \\
\hline
\end{tabular}

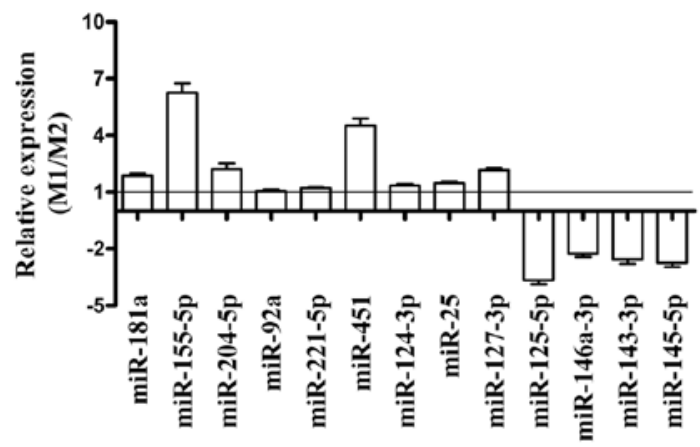

Figure 3. Confirmation of the differential expression of miRNAs using qRT-PCR. BMDMs were cultured in the presence of IFN- $\gamma(20 \mathrm{ng} / \mathrm{ml})$ plus LPS (100 ng/ml) or IL-4 (20 ng/ml). Relative expression folds of selected miRNAs were determined by TaqMan miRNA assays after normalization to U6 snRNA. Data are representative of three separate experiments, and show the means \pm SEM.

responding to selected inflammatory conditions (19-23,27-33). In these experiments, a subset of miRNAs was repeatedly found to be induced following inflammation-inducing stimuli, which caused induced differentiation towards the M1 phenotype. We hypothesized that miRNAs are involved, not only in macrophage responses to inflammatory conditions, but also in the modifications of gene expression required to generate a spectrum of macrophage activation patterns.

The aim of this study was to identify miRNAs that respond to stimuli inducing two different patterns of macrophage activation (M1 and M2). We profiled the expression of a number of miRNAs using miRNA microarray and demonstrated that the expression of 109 miRNAs was significantly different in M1 compared with M2. Using function classification, we were able to correlate the upregulation and downregulation of miRNAs to the inflammation-related processes, such as apoptosis, cell differentiation, cell proliferation, immune response, and inflammatory response. However, few regulatory pathways have been experimentally validated, the functional confirmation of which is to be confirmed in subsequent studies.

Figure 2. Clustering analysis of differential miRNA expression in macrophages with distinct polarizing conditions. BMDMs were cultured in the presence of IFN- $\gamma(20 \mathrm{ng} / \mathrm{ml})$ plus LPS $(100 \mathrm{ng} / \mathrm{ml})$ or IL-4 $(20 \mathrm{ng} /$ $\mathrm{ml})$. Clustering was performed to visualize the correlations among the replicates and varying sample conditions. The same amount of total RNA was used on each slide, thus the hybridization signals were normalized according to the total RNA concentration. Upregulated and downregulated genes are shown as red and green, respectively. M0, BMDMs; M1, BMDMs were cultured in the presence of IFN- $\gamma(20 \mathrm{ng} / \mathrm{ml})$ plus LPS $(100 \mathrm{ng} / \mathrm{ml})$; M2, BMDMs were cultured in the presence of IL-4 $(20 \mathrm{ng} / \mathrm{ml})$. 
Table II. microRNAs differentially expressed in polarized macrophages (M1 vs. M2, expression fold >2).

\begin{tabular}{|c|c|c|c|}
\hline \multicolumn{3}{|c|}{ Upregulated } & \multirow{2}{*}{$\frac{\text { Downregulated }}{\text { mmu-miR-125b-5p }}$} \\
\hline mmu-miR-155-5p & mmu-miR-3091-3p & mmu-miR-410-3p & \\
\hline mmu-miR-451 & mmu-miR-301b-5p & mmu-miR-544-3p & mmu-miR-466f-5p \\
\hline mmu-miR-363-5p & mmu-miR-153-5p & mmu-miR-675-5p & mmu-miR-466h-3p \\
\hline mmu-miR-3100-5p & mmu-miR-33-3p & mmu-miR-1932 & mmu-miR-145-5p \\
\hline mmu-miR-5617-3p & mmu-miR-542-5p & mmu-miR-1197-5p & mmu-miR-143-3p \\
\hline mmu-miR-7b-5p & mmu-miR-1930-5p & mmu-miR-1969 & mmu-miR-574-5p \\
\hline mmu-miR-448-3p & mmu-miR-3109-3p & mmu-miR-3970 & \\
\hline mmu-miR-1947-5p & mmu-miR-1298-5p & mmu-miR-5709 & \\
\hline mmu-miR-141-3p & mmu-miR-344d-2-5p & mmu-miR-3095-5p & \\
\hline mmu-miR-1950 & mmu-miR-714 & mmu-miR-5617-5p & \\
\hline mmu-miR-2139 & mmu-miR-3086-3p & mmu-miR-1955-3p & \\
\hline mmu-miR-3093-3p & mmu-miR-465a-5p & mmu-miR-223-5p & \\
\hline mmu-miR-883a-3p & mmu-miR-221-5p & mmu-miR-191-3p & \\
\hline mmu-let-7f-1-3p & mmu-miR-381-3p & mmu-miR-181d-3p & \\
\hline mmu-miR-2861 & mmu-miR-551b-5p & mmu-miR-152-5p & \\
\hline mmu-miR-202-3p & mmu-miR-212-5p & mmu-miR-683 & \\
\hline mmu-miR-5105 & mmu-miR-301a-5p & mmu-miR-344b-5p & \\
\hline mmu-miR-880-3p & mmu-miR-33-5p & mmu-miR-205-3p & \\
\hline mmu-miR-1953 & mmu-miR-379-3p & mmu-miR-453 & \\
\hline mmu-miR-691 & mmu-miR-3067-3p & mmu-miR-432 & \\
\hline mmu-miR-146a-3p & mmu-miR-1199-5p & mmu-miR-291b-5p & \\
\hline mmu-miR-876-3p & mmu-miR-592-3p & mmu-miR-190b-3p & \\
\hline mmu-miR-431-3p & mmu-miR-433-5p & mmu-miR-409-3p & \\
\hline mmu-miR-1948-5p & mmu-miR-491-5p & mmu-miR-652-5p & \\
\hline mmu-miR-127-3p & mmu-miR-3079-3p & mmu-miR-421-3p & \\
\hline mmu-miR-1897-3p & mmu-miR-3104-3p & mmu-miR-450a-2-3p & \\
\hline mmu-miR-291a-5p & mmu-miR-410-5p & mmu-miR-3080-3p & \\
\hline mmu-miR-1298-3p & mmu-miR-1955-5p & mmu-miR-876-5p & \\
\hline mmu-miR-471-3p & mmu-miR-200b-3p & mmu-miR-181a-1-3p & \\
\hline mmu-miR-204-5p & mmu-miR-804 & mmu-miR-551b-3p & \\
\hline mmu-miR-5626-5p & mmu-miR-3082-3p & mmu-miR-299-5p & \\
\hline mmu-miR-23b-5p & mmu-miR-122-3p & mmu-miR-136-5p & \\
\hline mmu-miR-124-3p & mmu-miR-205-5p & mmu-miR-128-1-5p & \\
\hline mmu-miR-3106-3p & mmu-miR-92a-1-5p & mmu-miR-3094-3p & \\
\hline mmu-miR-25-5p & mmu-miR-107-5p & & \\
\hline
\end{tabular}

Table III. Functional categories of differentially expressed microRNAs strictly associated with macrophage polarization.

\begin{tabular}{ll}
\hline Functions & \multicolumn{1}{c}{ microRNAs } \\
\hline Apoptosis & miR-181a, miR-155, miR-204, miR-92a, miR-146a, miR-221 \\
Cell differentiation & miR-145, miR-155, miR-143, miR-127 \\
Cell proliferation & miR-125b, miR-451, miR-145, miR-143, miR-124, miR-221, miR-92a \\
Immune response & miR-25, miR-125b, miR-181a, miR-223, miR-155, miR-143, miR-146a, miR-92a \\
Inflammation & miR-25, miR-125b, miR-181a, miR-223, miR-155, miR-146a, miR-143 \\
\hline
\end{tabular}

According to the function analysis of differentially expressed miRNAs, 13 of the 109 miRNAs were selected and qRT-PCR was used to confirm the results of the miRNA microarray analysis. In total, 9 of the 13 miRNAs tested were 
identified as being differentially expressed in $\mathrm{M} 1$ by the microarray and qRT-PCR. Similarities between our microarray data and those of previous studies have been identified. Previous studies have shown that several miRNAs, including miR-29b, miR-146a, miR-155, miR-193b, miR-222 and miR-125b, were elevated during monocytic cell differentiation towards macrophages $(21,23,33-35)$. These miRNAs appeared to be regulated in monocyte-derived macrophages (MDMs). In our study, in addition to miR-146a, miR-155 and miR-125b, high levels of miR-181a, miR-204-5p, miR-451 and miR-127-3p in M1 cells, and miR-143-3p and miR-145-5p in M2 cells were detected. Although there were discrepancies in the results of the microarray and the qRT-PCR analyses, the miRNA microarray provided a rapid method for identifying a large number of differentially expressed miRNAs in M1, which was confirmed by qRT-PCR.

This study has examined the global expression patterns of miRNAs in macrophage polarization and contributed to the growing understanding of the role of miRNAs in macrophage exposure to different activating conditions. Thus, miRNA profiling reveals novel molecules and signatures associated with differentiation of BMDMs and polarized activation which may be candidate targets in pathophysiology.

\section{Acknowledgements}

This study was financially supported by the Natural Science Foundation of the Anhui Higher Education Institutions of China (KJ2011B191, KJ2011B198) and the Foundation of Introducing Talents of Yijishan Hospital (YJRC2009003).

\section{References}

1. Rosa A, Ballarino M, Sorrentino A, et al: The interplay between the master transcription factor PU1 and miR-424 regulates human monocyte/macrophage differentiation. Proc Natl Acad Sci USA 104: 19849-19854, 2007.

2. Androulidaki A, Iliopoulos D, Arranz A, et al: The kinase Akt1 controls macrophage response to lipopolysaccharide by regulating microRNAs. Immunity 31 220-231, 2009.

3. Bazzoni F, Rossato M, Fabbri M, et al: Induction and regulatory function of miR-9 in human monocytes and neutrophils exposed to proinflammatory signals. Proc Natl Acad Sci USA 106: 5282-5287, 2009.

4. Du CS, Liu C, Kang JH, et al: MicroRNA miR-326 regulates $\mathrm{TH}-17$ differentiation and is associated with the pathogenesis of multiple sclerosis. Nat Immunol 10: 1252-1259, 2009.

5. Chang CI, Liao JC and Kuo L: Arginase modulates nitric oxide production in activated macrophages. Am J Physiol 274 H342-H348, 1998.

6. Chen T, Huang Z, Wang L, Wang Y, Wu F, Meng S and Wang C: MicroRNA-125a-5p partly regulates the inflammatory response lipid uptake and ORP9 expression in oxLDL-stimulated monocyte/macrophages. Cardiovas Res 83: 131-139, 2009.

7. Cheng Y,Kuang WH,Hao YH, et al: Downregulation of miR-27a* and miR-532-5p and upregulation of miR-146a and miR-155 in LPS-induced RAW2647 macrophage cells. Inflammation 35: 1308-1313, 2012.

8. Coley W, Van Duyne R, Carpio L, et al: Absence of DICER in monocytes and its regulation by HIV-1. J Biol Chem 285 31930-31943, 2010.

9. Dalton DK, Pitts-Meek S, Keshav IS, Figari A, Bradley A and Stewart TA: Multiple defects of immune cell function in mice with disrupted interferon-gamma genes. Science 259: 1739-1742, 1993.

10. Forrest AR, Kanamori-Katayama M, Tomaru Y, et al: Induction of microRNAs mir-155 mir-222 mir-424 and mir-503 promotes monocytic differentiation through combinatorial regulation. Leukemia 24: 460-466, 2010.
11. Goerdt S and Orfanos CE: Other functions other genes: alternative activation of antigen-presenting cells. Immunity 10 : 137-142, 1999.

12. Goerdt S, O Politz K, Schledzewski R, et al: Alternative versus classical activation of macrophages. Pathobiology 67: 222-226, 1999.

13. Gordon S: Alternative activation of macrophages. Nat Rev Immunol 3: 23-35, 2003.

14. Graff JW, Dickson AM, Gwendolyn C, McCaffrey AP and Wilson ME: Identifying functional microRNAs in macrophages with polarized phenotypes. J Biol Chem 287: 21816-21825, 2012.

15. Lewis BP, Burge CB and Bartel DP: Conserved seed pairing often flanked by adenosines indicates that thousands of human genes are microRNA targets. Cell 120: 15-20, 2005.

16. Liu G, Friggeri A, Yang Y, Park YJ, Tsuruta Y and Abraham E: miR-147 a microRNA that is induced upon Toll-like receptor stimulation regulates murine macrophage inflammatory responses. Proc Natl Acad Sci USA 106: 15819-15824, 2009.

17. Lu M, Shi B, Wang J, Cao Q and Cui QH: TAM: a method for enrichment and depletion analysis of a microRNA category in a list of microRNAs. BMC Bioinformatics 11: 419-426, 2010.

18. Lumeng CN, Bodzin JL and Saltiel AR: Obesity induces a phenotypic switch in adipose tissue macrophage polarization. J Clin Invest 117: 175-184, 2007.

19. Mallory AC and Vaucheret H: MicroRNAs: something important between the genes. Curr Opin Plant Biol 7: 120-125, 2004.

20. Mantovani A, Sica A and Locati M: Macrophage polarization comes of age. Immunity 23: 344-346, 2005.

21. Mantovani A, Sica A, Sozzani S, Allavena P, Vecchi A and Locati M: The chemokine system in diverse forms of macrophage activation and polarization. Trends Immunol 25: 677-686, 2004.

22. Mantovani A, Sozzani S, Locati M, Allavena P and Sica A: Macrophage polarization: tumor-associated macrophages as a paradigm for polarized M2 mononuclear phagocytes. Trends Immunol 23: 549-555, 2002.

23. Mills CD, Kincaid K, Alt JM, Heilman MJ and Hill AM: M-1/M-2 macrophages and the Th1/Th2 paradigm. J Immunol 164: 6166-6173, 2000.

24. Monk CE, Hutvagner G and Arthur JS: Regulation of miRNA transcription in macrophages in response to Candida albicans. PLoS One 5: e13669, 2010.

25. Mosser DM: The many faces of macrophage activation. J Leukoc Biol 73: 209-212, 2003.

26. Mosser DM and Edwards JP: Exploring the full spectrum of macrophage activation. Nat Rev Immunol 8: 958-969, 2008.

27. Munder M, Eichmann K and Modolell M: Alternative metabolic states in murine macrophages reflected by the nitric oxide synthase/arginase balance: competitive regulation by $\mathrm{CD}^{+}$ $\mathrm{T}$ cells correlates with Th1/Th2 phenotype. J Immunol 160: 5347-5354, 1998.

28. Nathan CF, Murray HW, Wiebe ME and Rubin BY: Identification of interferon-gamma as the lymphokine that activates human macrophage oxidative metabolism and antimicrobial activity. J Exp Med 158: 670-689, 1983.

29. O'Connell RM, Taganov KD, Boldin MP, Cheng G and Baltimore D: MicroRNA-155 is induced during the macrophage inflammatory response. Proc Natl Acad Sci USA 104: 1604-1609, 2007.

30. O'Connell RM, Kahn D, Gibson WS, et al: MicroRNA-155 promotes autoimmune inflammation by enhancing inflammatory T cell development. Immunity 33: 607-619, 2010.

31. Ruggiero T, Trabucchi M, De Santa F, et al: LPS induces $\mathrm{KH}$-type splicing regulatory protein-dependent processing of microRNA-155 precursors in macrophages. FASEB J 23: 2898-2908, 2009.

32. Schulte LN, Eulalio A, Mollenkopf HJ, Reinhardt R and Vogel J: Analysis of the host microRNA response to Salmonella uncovers the control of major cytokines by the let-7 family. EMBO J 30: 1977-1989, 2011.

33. Taganov KD, Boldin MP, Chang KJ and Baltimore D: NF-kappaBdependent induction of microRNA miR-146 an inhibitor targeted to signaling proteins of innate immune responses. Proc Natl Acad Sci USA 103: 12481-12486, 2006.

34. Tili E, Michaille JJ, Cimino A, et al: Modulation of miR-155 and miR-125b levels following lipopolysaccharide/TNF-alpha stimulation and their possible roles in regulating the response to endotoxin shock. J Immunol 179: 5082-5089, 2007.

35. Tserel L, Runnel T, Kisand K, et al: MicroRNA expression profiles of human blood monocyte-derived dendritic cells and macrophages reveal $\mathrm{miR}-511$ as putative positive regulator of Toll-like receptor 4. J Biol Chem 286: 26487-26495, 2011. 